\title{
Sepsis due to Vibrio alginolyticus isolated from catheter of young patient with hypercholesterolemy: the first case from Turkey
}

\author{
Lutfiye Oksuz, Nezahat Gurler \\ Istanbul University, Istanbul Faculty of Medicine, Department of Medical Microbiology, Capa-Istanbul-Turkey
}

Email address:

loksuz34@yahoo.com(L. Oksuz),ngurler@istanbul.edu.tr(N. Gurler)

To cite this article:

Lutfiye Oksuz, Nezahat Gurler. Sepsis due to Vibrio Alginolyticus Isolated from Catheter of Young Patient with Hypercholesterolemy: the First Case from Turkey. Clinical Medicine Research. Vol. 2, No. 3, 2013, pp. 37-39.doi: 10.11648/j.cmr.20130203.14

\begin{abstract}
Vibrio alginolyticus occasionally causes life-threatening infections in immunocompromised individuals. Bacteremia and necrotising fasciits caused by V.alginolyticus have been reported. We described a case of sepsis due to V. alginolyticus isolated from catheter of 19-year-old patient with hypercholesterolemy. Moreover the cases of invasive V.alginolyticus reported in the literature were reviewed. Blood cultures of the patient were evaluated by the BACTEC 9120 system. The identification of the bacteria was performed conventional methods and confirmed by the automatized ID32GN and VITEK 2 automation systems. Antibiotic susceptibility tests were performed according to recommendations of Clinical Laboratory and Standards Institute (CLSI). The patient recovered due to early diagnosis and appropriate therapy. No identifiable source of this infection could be determined. In conclusion, rapid and correct identification of the bacteria and early administration of appropriate antibiotics is essential for controlling invasive Vibrio infections, such as V.alginolyticus sepsis, especially in immunocompromised hosts. To the best of our knowledge, this is the first report of V.alginolyticus bacteremia in Turkey.
\end{abstract}

Keywords: Vibrio alginolyticus, Bacteremia, Sepsis, Hypercholesterolemy, Turkey

\section{Introduction}

Vibrios have been recognized as human pathogens, and they can be acquired through either ingestion of contaminated seafood or contact of traumatized skin with seawater(1). Extraintestinal Vibrio infections are most commonly cutaneus wounds or otitis externa, where breaks in the skin have become contaminated while swimming or boating in infected marine waters or after handling contaminated raw seafood. Most clinical isolates are recovered from superficial wounds or the external ears (2). Other Vibrio cholera, less common halophilic Vibrio spp include V.vulnificus, V.alginolyticus, V.fluvialis, Vhollisae, V.damsale. Molecular methods can be suggested for identification or detection of these species (15).

V.alginolyticus, first identified as a pathogen of humans in 1973, has been predominantly associated with cellulitis and acute otitis media or externa rather than gastroenteritis $(1,14)$. V.alginolyticus occasionally causes life-threatening infections in immunocompromised individuals (5). Conjunctivitis, acute gastroenteritis, bacteremia and necrotising fasciits caused by Valginolyticus have also been reported (2). Bacteremic infections of Valginolyticus are severe and occure only in patients with underlying diseases (12).

We described a case of sepsis due to V. alginolyticus isolated from catheter of 19-year-old patient with hypercholesterolemy in this paper. To the best of our knowledge, this is the first report case of V.alginolyticus bacteremia in Turkey.

\section{Case}

A 19-year-old patient was admitted to Department of Pediatric Cardiology, Istanbul Faculty of Medicine with high fever about ten days before admission although he was given amoxycillin+clavulanate. He was applied routinally lipid aferesis because of diagnosed with genetic hypercholesterolemy Type $2 \mathrm{a}$ while he was seven years old. He was being followed by Nutrition and Metabolism Department. A catheter had inserted to him before one and a half years.

The patient presented with high fever $\left(39^{\circ} \mathrm{C}\right)$ on admission. Blood cultures were obtained from the catheter and peripheral vein. After three days, the result of blood cultures was reported as V.alginolyticus. At the same time, the situation of the patient was deteriorated, by increase of C-reactive protein (CRP: $103 \mathrm{mg} / \mathrm{dl}$ ) and went to sepsis. He had emprically started on meropenem and teicoplanin before blood culture result was 
available. Blood cultures were taken from the catheter and from a peripheral vein again. The same bacteria was isolated from blood culture obtained catheter again. According to blood culture result, he continued on meropenem I.V. 1 g (3x1) therapy. CRP of the patient decreased (CRP: $14 \mathrm{mg} / \mathrm{dl}$ ). The patient recovered and discharged after 17 days. No identifiable source of this infection could be determined.

\section{Materials and Methods}

Blood cultures were evaluated on the BACTEC 9120 system (Beckton Dickinson, USA). After positive signal, the blood samples were inoculated sheep-blood and chocolated agar (bioMerieux, France). When the colonies were seen on sheep blood agar, they're subcultured on thiosulfate-citrate-bile salts-sucrose (TCBS) agar. The identification of the bacteria were performed by conventional methods and confirmed by the API ID 32 GN system (bioMerieux, France) and by the VITEK 2 system (bioMerieux, France). Antibiotic susceptibility tests were performed by standard disc diffusion and by E-test method according to the recommendations of Clinical and Laboratory Standards Institute (CLSI) (6).

Table 1. Some biochemical characteristics of V.alginolyticus isolate.

\begin{tabular}{|c|c|}
\hline Test & Reaction \\
\hline Growth on sheep blood agar (swarming) & Positive \\
\hline Growth on Mac Conkey agar & Positive \\
\hline \multicolumn{2}{|l|}{ Growth in Nutrient Broth with: } \\
\hline $0 \% \mathrm{NaCl}$ & Negative \\
\hline $1 \% \mathrm{NaCl}$ & Positive \\
\hline $6 \% \mathrm{NaCl}$ & Positive \\
\hline $8 \% \mathrm{NaCl}$ & Positive \\
\hline $10 \% \mathrm{NaCl}$ & Positive \\
\hline Motility & Positive \\
\hline Cytochrome oxidase & Positive \\
\hline Catalase & Positive \\
\hline \multicolumn{2}{|l|}{ Carbohydrate fermentation: } \\
\hline Glucose & Positive \\
\hline Lactose & Negative \\
\hline Mannose & Positive \\
\hline Mannitol & Positive \\
\hline Maltose & Positive \\
\hline Sucrose & Positive \\
\hline Ribose & Positive \\
\hline Sorbitol & Negative \\
\hline Rhamnose & Negative \\
\hline Inositol & Negative \\
\hline Nirate reduction & Positive \\
\hline Simmons citrate & Negative \\
\hline Indole & Positive \\
\hline Voges-Proskauer & Positive \\
\hline Lysine decarboxylase & Positive \\
\hline Ornithine decarboxylase & Positive \\
\hline Arginine decarboxylase & Negative \\
\hline L-alanine & Negative \\
\hline L-arabinose & Negative \\
\hline Hydrogen sulfide & Negative \\
\hline Esculin & Negative \\
\hline Gas production & Negative \\
\hline Urea hydrolysis & Negative \\
\hline Alkalen phosfatase & Positive \\
\hline $\mathrm{N}$-acetyl glucosamine & Positive \\
\hline Glycogen & Positive \\
\hline
\end{tabular}

\subsection{Microbiology}

While blood culture obtained from the peripheral vein remained negative, a positive signal was taken on the blood culture bottle from the cathether. Gram negative rods were seen in Gram stain prepared from positive blood culture bottle. After 24 hours of incubating the culture-positive samples, straight or curved, Gram-negative, oxidase-positive colonies were seen on sheep-blood and chocolated agar. The colonies were smooth, convex, creamy in consistency, and gray-white with full margins. Yellow colonies were seen on TCBS agar after 24 hours of incubation. Further identification was confirmed with the following biochemical tests. The strain showed a positive reaction to indol production, Voges-Proskauer, lysine decarboxylase, and ornithine dihydrolase, glucose fermentation, gelatin hydrolysis, reduction from nitrate to nitrite, alkalen phosfatase and growth in Nutrient broth with $6-10 \% \mathrm{NaCl}$. Negative findings included: growth on Simmons' citrate, growth in Nutrient broth with $0 \% \mathrm{NaCl}, \mathrm{H} 2 \mathrm{~S}$ on tri-sugar iron agar (TSI), gas production, urea hydrolysis, and arginine decarboxylase, myo-inositol, lactose fermentation. Some biochemical characteristics of the bacteria were showed in Table 1.

Table 2. The results of antibiotic susceptibility test of V.alginolyticus isolate. The disc diffusion method to some antibiotics for antimicrobial susceptibility testing of this organism has not been standardized, although the method is used.

\begin{tabular}{ll}
\hline Antibiotics & Susceptibility \\
\hline Ampicillin & Resistant \\
Cephazolin & Susceptible \\
Cefuroxime & Susceptible \\
Cefotaxime & Susceptible \\
Imipenem & Susceptible \\
Meropenem & Susceptible \\
Chloramphenicol & Susceptible \\
Trimethoprim-sulfametoxazole & Susceptible \\
Gentamycin & Susceptible \\
Tobramycin & Susceptible \\
Amikacin & Susceptible \\
Ciprofloxacin & Susceptible \\
Colistin & Susceptible \\
Erithromycin & Susceptible \\
\hline
\end{tabular}

The string test of the colonies was positive. In the string test, which lyses the vibrio cells, but not those of Aeromonas spp and Plesiomonas shigelloides, the strain was emulsified in $0.5 \%$ sodium deoxycholate (3). The bacteria were identified as $V$. alginolyticus by the API ID 32 GN system (bioMerieux, France) at a $95.3 \%$ confidence level and by the VITEK 2 system (biomerieux, France) at a 93\% confidence level. $V$. alginolyticus was isolated from the cathether while blood culture obtained from the peripheral vein remained sterile.

The organism was susceptible to a variety of antibiotics including cephazolin, cefuroxime, cefotaxime, gentamicin, trimethoprim-sulfamethoxazole, ciprofloxacin, but ampicillin resistant. The results of antibiotic susceptibility testing were showed in Table 2. 


\section{Discussion}

V.alginolyticus is a curved, motile, halophilic, Gram negative bacillus that is considered part of normal marine flora (4). The organism derived its name from its ability to utilize alginate. This species is the most salt-tolerant among the pathogenic Vibrio species and can even grow in extremely high salt concentrations (as high as 10\%). V.alginolyticus is considered relatively non-pathogenic in humans. The reason for Valginolyticus's lack of virulence remains unclear. Its virulence is related to its ability to produce hemolysis, hemagglutination and protease $(5,14)$. V.vulnificus and Valginolyticus are more associated with soft tissue wound infection and sepsis(15). Isolation of Valginolyticus is similar to that for V.vulnificus; however, V.alginolyticus does not ferment lactose (1).

V.alginolyticus has rarely been associated with respiratory tract infections and bacteremia, illnesses that may result in death in immunocompromised patients. In contrast to bacteremia caused by V.vulnificus, a highly invasive Vibrio species, sepsis has rarely been attributed to Valginolyticus $(4,15)$.

It has been rarely reported that Valginolyticus causes deep-seated or invasive infections. V.alginolyticus sepsis has lower pathogenicity, compared with $V$. vulnificus. The relative mortality of Valginolyticus sepsis is also lower than that of $V$. vulnificus (5).

In the search of the PUBMED database with keywords "Valginolyticus bacteremia", we found few cases of bacteremia caused by V.alginolyticus. The similar features of these patients were their predisposing factors such as cirrhosis, cancer, hemodialysis, burn, leukemia, sarchoma, chronic osteomyelitis and streoid therapy $(4,5,7,8,10,11)$. The patient who get routinally lipid apheresis in this report, had genetic hypercholesterolemy Type $2 \mathrm{a}$ and a catheter had inserted to him before one and a half years. His genetic diagnosis, implementation of apheresis and inserted catheter were predisposing factors of this patient. The patient recovered due to early diagnosis and appropriate therapy.

Vibrio species are usually resistant to penicillins and vancomycin, but are susceptible to tetracycline, chloramphenicol, aminoglicosides and the other B-lactam antibiotics(13). Tetracycline treatment usually results in cure(14). V.alginolyticus strain in this report was found to be resistant to penicillin, ampicillin and trimethoprim-sulfametoxazole but susceptible to the other antibiotics.

In conclusion, rapid and correct identification of the bacteria and early administration of appropriate antibiotics is crucial for controlling invasive Vibrio infections, such as Valginolyticus sepsis, especially in immunocompromised hosts.

\section{References}

[1] Neill MA, Carpenter CCJ. Other pathogenic vibrios. "Mandell GL, Bennett JE, Dolin R (eds): Principles and Practice of
Infectious Diseases" $7^{\text {th }}$ edition, Vol 2, p.2787, Philadelphia, USA, 2010.

[2] Winn W, Allen S, Janda W, Koneman E, Procop G, Schreckenberger P, Wood G (eds): "Koneman's Color Atlas and Textbook of Diagnostic Microbiology, $6^{\text {th }}$ edition". Curved Gram negative bacilli and oxidase positive fermenter: Campylobacteriaceae and Vibrionaceae. Chapter 8, s.393-428, Lippincott Williams\&Wilkins, Baltimore, USA, 2006.

[3] Forbes BA, Sahm DF, Weissfeld AS (eds):" Bailey\&Scott's Diagnostic Microbiology, $11^{\text {th }}$ edition", Chapter 31. Vibrio, Aeromonas, Plesiomonas and Chromobacterium violaceum, $\mathrm{p}$. 423-33, Mosby, USA, 2002.

[4] Chien JY, Shih JT, Hsueh PR, Yang PC, Luh KT. V.alginolyticus as the cause of pleural emptema and bacteremia in an immunocompromised patient. Eur J Clin Microbiol Infect Dis 2002, 21: 401-403.

[5] Lee DY, Moon SY, Lee SO, Young HY, Lee HJ, Lee MS. Septic shock due to V.alginolyticus in a cirrhotic patient: The first case in Korea. Yonsei Med J 2008, 49 (2): 329-32.

[6] Clinical and Laboratory Standards Institute (CLSI). Performance Standards for Antimicrobial Suscebtibility Testing. $17^{\text {th }}$ Informational Supplement. M100-S17, Vol 27, No 1, Table 2I, p.133, Wayne, PA: Clinical and Laboratory Standards Institute, 2007.

[7] Ruiz CC, Agraharkar M. Unusual marine pathogens causing cellulitis and bacteremia in hemodialysis patients: report of two cases and review of the literature. Hemodial Int. 2003:7(4):356-9

[8] Robert R, Grollier G, Malin F, Doré P, Pourrat O. Isolation of Vibrio alginolyticus from blood cultures in a leukaemic patient after consumption of oysters. Eur J Clin Microbiol Infect Dis. 1991;10(11):987-8.

[9] Barbarossa V, Kucisec-Tepes N, Aldova E, Matek D, Stipoljev F. Ilizarov technique in the treatment of chronic osteomyelitis caused by Vibrio alginolyticus. Croatian Medical Journal 2002, 43(3): 346-349

[10] English VL, Lindberg RB. Isolation of Vibrio alginolyticus from wounds and blood of a burn patient. American Journal of Medical Technology 1977, 43(10): 989-993

[11] Janda JM, Brenden R, Debenedetti JA, et al. Vibrio alginolyticus bacteremia in an immunocompromised patient. Diagnostic Microb Infect Dis 1986, 5(4): 337-340

[12] Bonner JR, Coker AS, Berryman CR, Pollock HM. Spectrum of Vibrio Infections in a Gulf Coast Community. Ann Intern Med. 1983;99(4):464-469

[13] Schmidt U, Chmel H, Cobbs C. Vibrio alginolyticus infections in humans. J Clin Microbiol 1979;10:666-8.

[14] Waldor MK, Keusch GT. Cholera and other vibrioses. Kasper DL, Fauci AS (eds): Harrison's Infectious Diseases. 17 edition, Chapter 57, p. 540, McGraw Hill Companies, Inc, USA, 2010.

[15] Megraud F, Thijsen SFT. Curved and spiral bacilli. Cohen J, Powderly WG (eds): Infectious Diseases, $2^{\text {nd }}$ edition, Chapter 230, p. 2227, Elsevier Ltd, UK, 2004. 